\title{
EFEITOS DA AVALIAÇÃO SOBRE OS PROGRAMAS BRASILEIROS DE PÓS-GRADUAÇÃO EM ADMINISTRAÇÃO: ANÁLISE SOBRE O ISOMORFISMO
}

http://dx.doi.org/10.5902/2318133862676

\author{
Danieli Artuzi Pes Backes ${ }^{1}$ \\ Fernando Antonio Ribeiro Serra ${ }^{2}$ \\ Josiane de Oliveira Lobato ${ }^{3}$ \\ Sandra Negri ${ }^{4}$
}

\begin{abstract}
Resumo
O objetivo deste texto é apresentar resultados de pesquisa relacionada com os efeitos da avaliação da Capes sobre os programas de pós-graduação em Administração - PPGAs. O método inclui estatística descritiva, análise de agrupamentos, revisão bibliográfica e documental. Os resultados apontaram a presença de isomorfismo mimético entre os PPGAs com diferentes intensidades. O maior grau de isomorfismo entre os grupos mais homogêneos torna evidente a disseminação da prática de espelhamento nas propostas curriculares, em especial nos programas menos tradicionais. Observou-se nos últimos anos que a Capes, que ora contribuiu para o isomorfismo, passou a adotar medidas para reduzir as similaridades, evitando o comprometimento evolutivo da ciência. As implicações do isomorfismo são a geração de entraves para os avanços qualitativos da produção científica.
\end{abstract}

Palavras-chave: avaliação; pressões institucionais; isomorfismo; Capes; programas de pósgraduação.

\section{EFFECTS OF THE EVALUATION ON BRAZILIAN POSTGRADUATE PROGRAMS IN ADMINISTRATION: ANALYSIS ON ISOMORPHISM}

\section{Abstract}

The objective was to verify the effects of the evaluation on the Graduate Programs in Administration - PPGAs. The method includes descriptive statistics, cluster analysis, bibliographic and document review. The results indicated the presence of mimetic isomorphism among PPGAs with different intensities. The greater degree of isomorphism among the most homogeneous groups is evident in the dissemination of the practice of mirroring in curricular proposals, especially in less traditional programs. It has been observed in recent years that Capes, which now contributed to the isomorphism, started to adopt measures to reduce similarities, avoiding the evolutionary compromise of science. Evidence of isomorphism is the generation of obstacles to qualitative advances in scientific production.

Key-words: evaluation; institutional pressures; isomorphism; Capes; graduate programs.

\footnotetext{
1 Universidade Federal de Mato Grosso, Brasil. E-mail: backes.dani@gmail.com.

2 Universidade Nove de Julho, São Paulo, Brasil. E-mail: fernandorserra@gmail.com.

3 Universidade Federal de Mato Grosso, Brasil. E-mail: josiane deoliveira@hotmail.com.

4 Universidade Federal de Mato Grosso, Brasil. E-mail: sandra.negri.br@gmail.com. 


\section{Introdução}

E mbora até a década de 1970 a avaliação institucional não fosse tão presente no ensino superior brasileiro, a partir de meados de então se iniciou o processo de avaliação sistemática dos cursos de pós-graduação stricto sensu, que se estendeu aos cursos superiores de graduação. O sistema de avaliação envolvia duas concepções, uma que primava pela avaliação emancipadora e formativa, baseada da aprendizagem institucional, e a segunda, que se firmava na regulação externa e estabelecimento de rankings calcados no desempenho organizacional (Guerra; Souza, 2020).

Os cursos de pós-graduação stricto sensu são avaliados pela Coordenação de Aperfeiçoamento de Pessoal de Nível Superior - Capes. Embora o sistema seja considerado um dos mais modernos do mundo (Maccari; Lima; Riccio; 2009; Nascimento; 2010), existem críticas, como a de que o sistema é padronizado para todas as áreas do conhecimento. Spagnolo e Calhau (2002) também apontam a heterogeneidade entre as diferentes áreas do conhecimento e a diversidade quanto as questões econômicas e de acesso aos recursos. O sistema avaliativo pode gerar pressões e incertezas porque compara os desempenhos por meio de um ranking (Maccari et al., 2009), sendo que o conceito obtido se reflete na capacidade de captação de recursos (Dias Sobrinho, 2003).

$\mathrm{Na}$ busca pela legitimação e melhores oportunidades de acesso aos recursos os programas de pós-graduação, em especial os que estão nas posições menos elevadas do ranking, podem optar por se espelhar nas estruturas e práticas dos programas mais tradicionais e melhor posicionados, sendo que um dos elementos observados podem ser os currículos. A adoção de currículos similares pode gerar isomorfismo. O fenômeno denominado isomorfismo ocorre em razão do nível de estruturação do campo organizacional (Peci, 2006) e pode ser de três tipos: coercitivo, normativo e mimético (Dimaggio; Powell, 1983).

A disposição em assemelhar a estrutura a outras instituições de forma intencional pode ser examinada pela abordagem do isomorfismo mimético, que diz respeito à imitação de processos, práticas e estrutura de outras organizações. Haveman (1993) defende que 0 isomorfismo mimético é a realização da conformidade pela imitação. $O$ isomorfismo mimético se estabelece com maior frequência num campo com elevada incerteza e pode estar associado ao desejo de se assemelhar às instituições mais bemsucedidas com a intenção de aumentar as chances de sucesso (Dimaggio; Powell, 1983; Almeida; Callado, 2017). Essa prática existe entre as organizações e tanto maior tende a ser quanto mais regulamentado o campo organizacional (Peci, 2006).

Estudos que constataram a presença de isomorfismo institucional já foram realizados nos cursos de graduação em Administração brasileiros, como o de Langrafe, Boaventura, Silva, da Silva (2009), e em cursos de pós-graduação, identificando clusters a partir de variáveis quantitativas (Backes; Serra; Zarour, 2018), todavia não foram encontradas pesquisas nas pós-graduações, o nível educacional superior mais regulamentado. Portanto, nesse estudo temos por objetivo identificar a presença de isomorfismo mimético a partir das similaridades estruturais dos currículos dos programas de pós-graduação em Administração de estrato superior - entre 4 e 7 -, ao mesmo tempo em que procuramos verificar se a participação nos agrupamentos determina seu posicionamento no ranking da Capes. 
A escolha desse campo organizacional é relevante porque no Brasil o curso de graduação em Administração é um dos maiores em número de alunos, sendo que até 2015 registrava o maior número de ingressantes e concluintes, se mantendo atualmente na segunda posição (Inep, 2018). Essa expansão se estende à pós-graduação, que em 2016 possuía 135 programas (Capes, 2018a), fazendo parte da segunda maior grande área da Capes em número de programas (Capes, 2018b).

\section{Isomorfismo mimético}

Segundo Meyer e Rowan (1977) "muitas estruturas organizacionais formais surgem como reflexos de regras institucionais racionalizadas" (p. 340) e essas regras, ao serem assimiladas pelas organizações, se tornam legitimadas, estabilizando-se, ampliando suas perspectivas de sobrevivência e acesso a recursos. Contudo, a absorção das regras institucionais pode tornar as organizações isomórficas. Há três tipos de isomorfismo: o coercitivo, o normativo e o mimético. $O$ isomorfismo coercitivo deriva das pressões de umas instituições sobre as outras, podendo ser de origem governamental, da relação de dependência com outras organizações ou de pressões e expectativas sociais. $O$ isomorfismo normativo provém da troca de informações entre profissionais por meio de associações e instituições profissionalizantes. O isomorfismo mimético é a imitação ou implantação do modelo de outras organizações e ocorre diante de incertezas, objetivos ambíguos e tecnologias mal compreendidas (Dimaggio; Powell, 1983).

No cenário onde não há clareza na relação entre meios e fins ou quando os objetivos organizacionais não estão bem definidos as organizações costumam observar o que outras estão fazendo, julgando se as ações das organizações estruturalmente equivalentes são adequadas (Han, 1994), e dessa forma, podem economizar com custos de pesquisa, substituindo as regras institucionais pelas regras técnicas (Haveman, 1993). Quando as organizações se encontram num campo bem estruturado onde existem pressões externas que variam desde avaliação, exames ou até outras formas de acreditação, tendem a agir defensivamente e atuar de maneira similar às outras organizações que conseguem obter sucesso, deixando o planejamento estratégico em segundo plano para atender às leis e normas de regulamentação (Maccari; De Almeida; Riccio; Alejandro, 2014). Conforme as pressões regulatórias aumentam as organizações elevam o grau de formalização de normas e procedimentos (Frumkin; Galaskiewicz, 2004), levando a mudanças cada vez mais isomórficas.

Diante das pressões exercidas pela Capes, que vão desde a normatização para criação de novos cursos até a avaliação periódica (Capes, 2017), acreditamos constatar a existência de isomorfismo mimético entre os programas de pós-graduação em Administração. Portanto, nossa primeira proposição é a de que:

P1: existe isomorfismo mimético entre os programas de pós-graduação stricto sensu em Administração no Brasil, decorrentes das pressões institucionais externas.

As ações de muitas organizações, incluindo as escolares, têm como base o quadro social do grupo ao qual pertencem (Han, 1994), por isso algumas estratégias são tão populares em alguns campos organizacionais (Pedersen; Neergaard; Pedersen; Gwozdz, 2013). Esse é o cenário que supomos encontrar no campo educacional dos programas de pós-graduação em Administração, pois os cursos de pós-graduação brasileiros, especialmente da área da Administração, seguiam modelos similares devido à forte

\begin{tabular}{|l|l|l|l|l|l|}
\hline Regae: Rev. Gest. Aval. Educ. & Santa Maria & v. 10 & n. 19 & e62676, p. 1-21 & 2021 \\
\hline
\end{tabular}


influência americana adotada desde o início da fundação dos programas (Santos; Azevedo, 2009), por volta de 1970. Esse fato pode ter gerado isomorfismo mimético, que se trata da intenção de assemelhar a própria estrutura à estrutura de outras organizações ou ainda seria um processo de mudanças baseadas em tendências compartilhadas entre organizações (Tolbert; Zucker, 1983; Burns; Wholey, 1993).

Todavia, os programas se encontram num ambiente altamente regulamentado por órgãos governamentais, como a Capes, que nos últimos anos adotou diretrizes para impedir a completa semelhança entre as estruturas e práticas. Atualmente a Capes possui um conjunto de normas para avaliação dos programas que incluem os critérios de inovação e relevância local, regional ou nacional para aprovação de novos cursos (Capes, 2016). Isso impede que todos os programas mantenham estruturas semelhantes, especialmente os mais jovens. Assim, mesmo pertencendo a um campo organizacional comum, esperamos encontrar similaridades e dissimilaridades entre os PPGAs, que poderão formar diferentes agrupamentos.

Assim, como segunda proposição sustentamos que P2: há similaridade entre as estruturas curriculares dos PPGAs que poderão formar agrupamentos específicos com características intraorganizacionais similares e características interorganizacionais dissimilares.

Nas últimas três décadas as instituições de ensino superior passaram por maior responsabilização e consequente obtenção de financiamento baseado no desempenho (Croucher; Woelert, 2016). A resposta foi a criação de sistemas de avaliação, que frequentemente são introduzidos com a lógica de aumentar a competitividade num mercado cada vez mais global de pesquisa na educação superior (Hammarfelt; Fredrik, 2015). Inevitavelmente essa dinâmica induz à competitividade entre as instituições, considerando que o desempenho mais elevado assegura maiores chances de acesso às melhores fontes de recursos. O estudo de Segev, Raveh e Farjoun (1999) encontrou resultados como a formação de seis grupos distintos, compostos pelas similaridades entre os 25 principais MBAs americanos, como resposta ao ambiente competitivo e, no Brasil, Langrafe, Boaventura, Silva, da Silva (2009) encontraram situação parecida entre os cursos de graduação em Administração das faculdades de São Paulo, identificando três tipos de agrupamentos, que concluíram serem formados a partir da adaptação às forças regulatórias. Da mesma forma Scafuto, Backes e Maccari (2017) constataram a presença de três grupos isomórficos entre os MBAs participantes do ranking da América Economia, verificando comportamentos complementares idiossincráticos entre as instituições e Backes, Serra e Zarour (2018) identificaram a formação de clusters entre programas de pós-graduação brasileiros a partir de variáveis quantitativas que se agruparam entre os programas pesquisados, que se atribuiu serem decorrentes de pressões externas do órgão de avaliação.

Tanto as forças regulatórias quanto os aspectos competitivos, que também podem ser decorrentes da regulamentação, conduzem as instituições a adotarem estratégias semelhantes na busca pela legitimação. $O$ reconhecimento e a legitimação podem vir a partir do bom desempenho na avaliação do órgão regulador, sendo que o fato de receber uma avaliação positiva significa maiores chances na captação de recursos (Dias Sobrinho, 2003). Assim, os gestores dos PPGAs tenderão a mover esforços para obter um bom posicionamento no ranking da Capes e uma das alternativas para reduzir as

\footnotetext{
Regae: Rev. Gest. Aval. Educ. $\quad$ Santa Maria
}

v. 10

ก. 19

e62676, p. 1-21

2021 
incertezas e aumentar as chances de sucesso, pode ser se espelhar em outra organização que presumidamente obteve êxito (Pedersen et al., 2013).

Portanto, a terceira proposição é P3: o pertencimento a um determinado agrupamento, formado a partir do isomorfismo estrutural, pode se refletir no desempenho do PPGA na avaliação da Capes.

Vários estudos foram realizados tendo como pano de fundo a abordagem do isomorfismo mimético. Dentre os mais citados se destacam Haveman (1993); Han (1994); Brouthers, O'Donnell e Hadjimarcou (2005); Tingling e Parent (2002); Benders, Batenburg e Van der Blonk (2006); Lu (2002); Appold (2005); Burns e Wholey (1993); Tolbert e Zucker (1983); Knoke (1982) e outros mais recentes são os de Yang e Hyland (2012), Darus, Hamzah e Yusoff, (2013); Martínez-Ferrero e García-Sánchez (2017); Savitri e Fanani (2017). Estudos que investigaram os efeitos do isomorfismo sobre as instituições de ensino superior foram os de Jarvis (2014); Hammarfelt e Fredrik (2015); Rossoni e de São Pedro Filho (2015); Croucher e Woelert (2016).

Metodologicamente, o manuscrito que serviu de base foi o de Segev, Raveh e Farjoun (1999), que buscaram identificar agrupamentos nas escolas de negócio norte americanas a partir da análise dos currículos, apontando as diferenças e similaridades destas na adaptação ao ambiente. Usando as mesmas variáveis, as disciplinas ou componentes curriculares, buscou-se identificar o comportamento isomórfico mimético entre os programas de pós-graduação em Administração, todavia a abordagem desta pesquisa utiliza método diferenciado.

\section{Procedimentos metodológicos}

Esse estudo é exploratório, de natureza quantitativa e se utilizou de estatística descritiva e análise de agrupamentos como método para coleta, apresentação e análise de dados. Quanto aos agrupamentos, por meio da classificação e categorização univariada, com base na análise do currículo dos programas de pós-graduação em Administração, foram formados grupos de PPGAs aglomerados pelas similaridades curriculares. A análise de agrupamentos é uma técnica para identificação de subgrupos mutuamente excludentes, a partir das similaridades entre indivíduos ou objetos. $\mathrm{Na}$ análise de agrupamentos os grupos não são predefinidos e a identificação faz parte da técnica. Para isso, são necessárias as definições dos critérios de agrupamento, que em geral consistem de três passos: a) definição da medida de similaridade, b) divisão dos indivíduos em grupos conforme as associações, c) determinação do perfil dos indivíduos para estabelecimento de sua composição (Hair; Anderson; Thatham; Black, 2005).

Embora os dados secundários coletados sejam textuais, o método não foi direcionado para análise de conteúdo (Bardin, 1977). Para a realização de análise de conteúdo seria necessária a revisão das ementas das disciplinas para verificação do seu teor (Quivy; Campenhoudt, 1998), porém, o objetivo do estudo foi segregar as disciplinas em eixos discriminantes de acordo com a compreensão de pertencimento à determinada área do conhecimento. Apesar do rigor e precisão das análises puramente quantitativas, elas deixam a desejar quanto ao critério da subjetividade mínima, que permite a exploração mais profunda do meio pesquisado (Quivy; Campenhoudt, 1998). 
Para atender aos objetivos os seguintes critérios foram adotados na coleta de dados:

a) Todas as informações para mapeamento dos programas foram coletadas na Plataforma Sucupira, no link referente à Coleta Capes - Disciplinas (Capes, 2017a).

b) Os programas incluídos no estudo são todos aqueles que possuem notas que estão no intervalo de 4 a 7 na avaliação trienal 2013 da Capes, no total de 37 . As notas selecionadas para a pesquisa certificam que a qualidade dos programas vai de boa, conceito 4, à excelência nacional, conceito 5, e excelência internacional, conceitos 6 e 7 (Capes, 2015).

c) os currículos dos programas passaram pela primeira triagem ainda na Plataforma Sucupira, sendo que as disciplinas que registraram duração inferior a um ano, denominação idêntica ou com a indicação de pertencerem a Minter ou Dinter foram eliminadas do estudo.

d) Programas com mais de uma centena de disciplinas cadastradas tiveram redução para no máximo cem unidades, tendo como critério de eliminação denominações redundantes e conteúdo semelhante em mais de um idioma, mantendo-se a nomenclatura em português.

Após a coleta e organização dos dados realizou-se a classificação das disciplinas por eixos, segundo um padrão geral, como por exemplo, Administração de Marketing ou Comportamento do Consumidor foram classificados como Marketing, ou Gestão de Pessoas, Comportamento Humano, Aprendizagem organizacional ou estudos organizacionais foram classificados como Organizações, e assim por diante. Após o encerramento da classificação iniciou-se a etapa de contagem da quantidade de disciplinas distribuídas entre os eixos de cada programa, conforme figura 3.

Quanto à análise de agrupamentos a técnica empregada foi a utilização dos filtros da planilha eletrônica, onde foram organizados os dados, cuja finalidade consistiu em segregar e comparar as estruturas dos cursos segundo os critérios que foram sendo adotados para formação dos grupos, sendo eles:

a) Programas que apresentavam similaridade em seis eixos, sendo considerados o grupo mais homogêneo. Os eixos selecionados foram Organizações, Métodos de Pesquisa, Marketing, Finanças, Estratégia e TI, Gestão de Sistemas e Inteligência. Essa sequência de eixos foi adotada por se tratar daqueles que apresentavam o maior número de programas vinculados.

b) Programas que apresentavam similaridade em cinco eixos, de modo que se aproveitou a seleção anterior sendo desconsiderados os eixos de trás para frente, nesse caso, TI, Gestão de Sistemas e Inteligência, e assim por diante até chegar aos dois primeiros eixos. Em todos os casos foram eliminados os resultados já captados no filtro anterior.

c) O próximo passo foi estabelecer eixos específicos para detectar similaridades entre os programas, sendo Sustentabilidade; Sociedade, Cultura e Mercado; Negócios e Empreendimentos Internacionais; TI, gestão de sistemas e Inteligência; Gestão Pública; Organizações. 
Após seleção de todos os eixos foi possível notar que os 37 programas haviam sido classificados, sendo encerrado o processo de busca. A próxima etapa foi a análise dos agrupamentos a partir da identificação das características comuns e similaridades estruturais.

\section{Resultados}

Os resultados estão apresentados em duas seções. A primeira se refere a caracterização dos PPGAs, segundo os currículos, e a segunda etapa apresenta a formação dos agrupamentos a partir das inferências realizadas em cada filtro.

\section{Caracterização dos currículos dos PPGAs}

Os programas de pós-graduação em Administração apresentam heterogeneidade quanto ao tamanho do currículo, interdisciplinaridade, diversidade e complexidade. A média da quantidade de disciplinas adotadas pelos programas é igual a 53,5 e a moda identificou dois valores comuns, sendo 42 e 36 componentes curriculares em três programas para cada uma das quantidades encontradas. O desvio-padrão é igual a 21,5 e a variância do conjunto de dados se mostrou elevada $(461,30)$, indicando que os tamanhos dos currículos estão dispersos em relação à média. A mediana, ou valor que divide o conjunto de dados em metade superior e metade inferior, é igual a 44, e a divisão do conjunto de dados pode ser observado na tabela 1.

Tabela 1 -

Divisão dos programas entre grades mais densas e grades mais enxutas.

\begin{tabular}{c|c|c|c|c|c|c|c}
\hline \multicolumn{3}{c}{ Programas com currículo mais denso } & \multicolumn{3}{c}{ Programas com currículo mais enxuto } \\
\hline UFRJ (43a) & 108 & Ufla (41A) & 70 & Uece (11A) & 43 & ESPM (6A) & 36 \\
\hline FGV/SP (42a) & 93 & UFPB/JP (40A) & 66 & Univali (13A) & 42 & UPM (17A) & 34 \\
\hline UFPE (21a) & 92 & PUC/PR(16A) & 63 & UFSM (13A) & 42 & FEI (9A) & 34 \\
\hline USP (41A) & 88 & UFPR (24a) & 60 & Unama (7A) & 42 & Unisinos (16a) & 33 \\
\hline UFMG (43a) & 79 & Uninove (10A) & 51 & UNB (40A) & 40 & Unir (10a) & 32 \\
\hline UFRN (38a) & 79 & UCS (10A) & 48 & Unigranrio (9A) & 40 & Udesc (16a) & 31 \\
\hline Ufrgs (44a) & 76 & PUC/RS (10A) & 45 & UFES (16A) & 38 & UFMS (8A) & 30 \\
\hline PUC/RJ(44a) & 75 & UEM (6A) & 45 & Furb (19A) & 37 & UFV (11A) & 28 \\
\hline FGV/RJ (49a) & 72 & USCS (13A) & 44 & PUC/SP (38a) & 36 & & \\
\hline UFSC (38a) & 71 & & & UFBA (33a) & 36 & & \\
\hline FonS das
\end{tabular}

Fonte: dados da pesquisa.

Conforme tabela 1 há tendência de currículos mais enxutos entre os programas mais jovens e mais amplos e diversificados entre os PPGAs mais antigos. Nesse estudo consideram-se programas antigos aqueles que possuem idade superior a 30 anos. Todavia, nem todo curso antigo tem currículos extenso, uma das exceções são os programas da UNB, PUC/SP e UFBA, ao mesmo tempo em que há casos, como o da UFPE, PUC/PR e UFPR dentre os PPGAs mais jovens, que possuem currículos bastante densos, sendo que $30 \%$ dos programas apresentam quantidade de componentes curriculares $30 \%$ acima da média, ao passo que $40 \%$ dos programas possuem currículos com quantidades em torno de $30 \%$ da média, para além ou para aquém, e $30 \%$ dos programas apresentam quantidade de componentes $30 \%$ abaixo da média. Esses valores

\begin{tabular}{|l|l|l|l|l|l|}
\hline Regae: Rev. Gest. Aval. Educ. & Santa Maria & v. 10 & n. 19 & e62676, p. 1-21 & 2021 \\
\hline
\end{tabular}


demonstram que a maioria dos cursos se concentra em torno da média obtida para o conjunto de dados, embora a diferença seja pequena para os que se distanciam e acabam por elevar o desvio-padrão.

Entretanto, a polarização em torno das quantidades de disciplinas não significa maior concentração ou maior amplitude em relação à diversidade de temas. Essa não é uma regra. Nem todo currículo enxuto é concentrado. Há muitos casos de currículos reduzidos e com disciplinas voltadas para as mais diversas áreas, chegando a dez diferentes categorias ou eixos disciplinares, como é o caso da UNB, Furb, UPM, Ufes, Udesc, Univali, USCS, UFSM, Uece, Unir, Unigranrio, Unama e UEM. Em compensação há currículos bastante focados, servindo como exemplo os programas da UFV e PUC/RS, com menos de sete eixos disciplinares cada um. Há currículos com alto nível de interdisciplinaridade - como é o caso da Ufrgs, UFPE, FGV/SP, Ufla, UFSC, PUC/SP, Ufes, Udesc, USCS, UFSM, UCS, Unir, FEI, Unama e UEM - mas se comparados ao total de programas estudados ainda representam uma parcela reduzida. Esse é um dos critérios valorizados pela Capes nas avaliações que, além da inovação incentiva a interdisciplinaridade nas propostas dos programas (Capes, 2016). Existe também grades com alto nível de complexidade na nomenclatura de seus componentes curriculares, os melhores exemplos são FGV/RJ e PUC/PR.

Os programas variam quanto à influência sobre o currículo dos demais PPGAs, se destacando nesse quesito alguns dos programas mais antigos. Destacaram-se como programas de maior referência os pertencentes à USP, FGV/SP, UFRJ e PUC/RJ, que podem ter influenciado de forma direta a estruturação curricular de vários programas. Dos 37 programas pesquisados 21 deles exerceram algum tipo de influência sobre os demais. Alguns programas apresentaram quantidade de relações de influência elevadas, todavia isso se explica porque aparentemente os programas costumam ter mais de um PPGA como modelo inspirador para sua grade. As relações individuais estabelecidas entre os programas de maior influência, conforme quantidade de eixos similares, estão dispostos nas figuras 1 e 2 . 
Figura 1 -

Relações da USP com outros PPGAs.

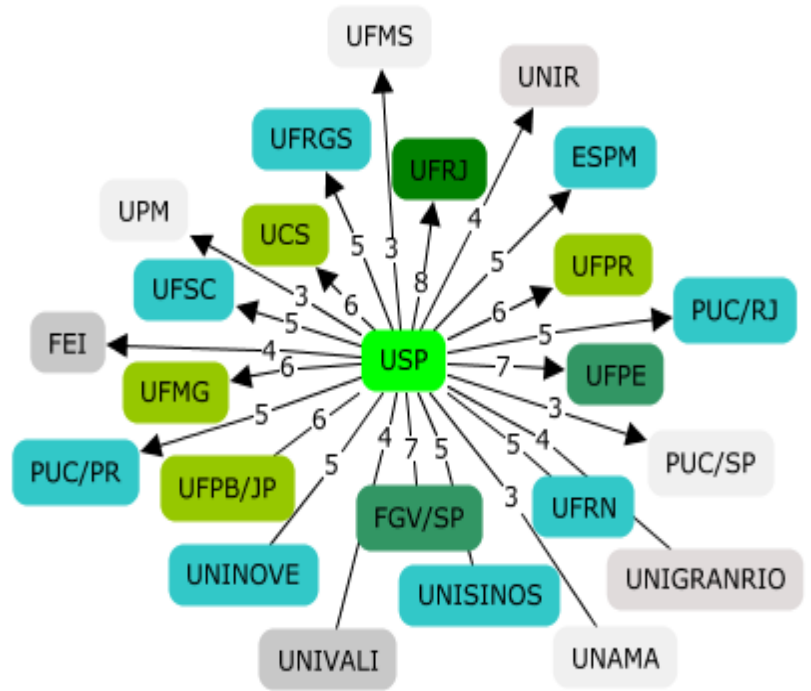

Figura 2 -

Relações FGV/SP com PPGAs.

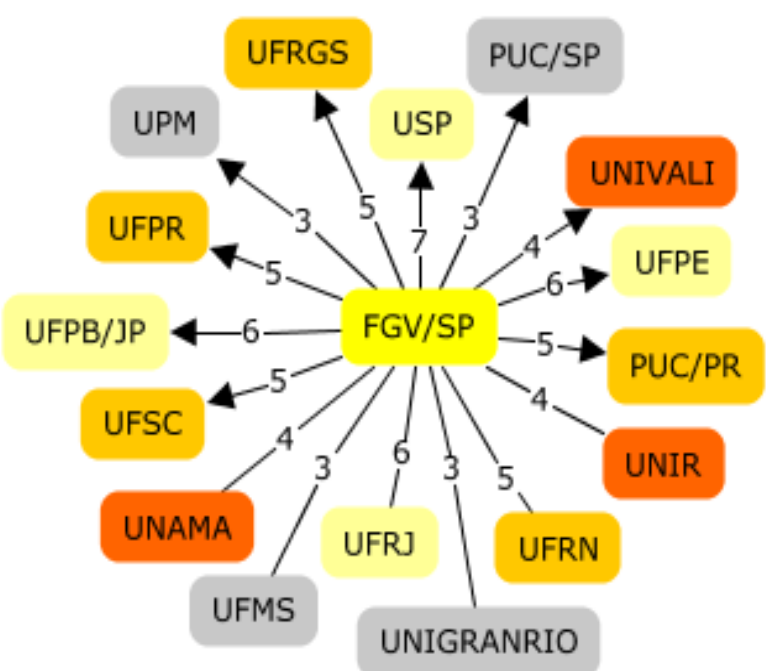

Fonte: dados da pesquisa.

Os valores expostos nas figuras 1 e 2 se tratam das quantidades de eixos comuns entre USP e FGV/SP e os outros programas, e não necessariamente a influência desses programas sobre os demais. Programas mais antigos e tradicionais, como os da Ufrgs, PUC/RJ, UFMG e UFRJ, poderiam não só ter se espelhado no currículo de ambas, como ter servido de modelo para elas. Já as relações com os programas mais jovens podem significar inspiração desses nas referidas instituições, pois segundo Dimaggio e Powell (1983) as organizações mais jovens e menos legitimadas podem decidir se assemelhar às organizações mais bem-sucedidas para aumentar as chances de sucesso.

Todavia, é importante notar que os currículos podem ter sido alterados pelos PPGAs ao longo do tempo e, nesse caso, a ordem cronológica não teria relevância, embora todo programa preserve suas competências essenciais ao atualizar os componentes curriculares. As core competences são aquelas competências, ou aquele conjunto de conhecimento, habilidades, tecnologias, sistemas físicos e gerenciais, difíceis de ser copiados, que geram valor percebido pela clientela e asseguram vantagem competitiva à organização (Prahalad; Hamel, 1990). Ademais, mesmo que os PPGAs possam alterar seus currículos a qualquer tempo, o completo alinhamento com a proposta do programa não permite tanta flexibilidade.

A seguir apresentamos a categorização das disciplinas em eixos relacionados às áreas de conhecimento da Administração. Com a figura 3 ilustra-se a classificação e a distribuição dos PPGAs após contagem e organização por eixos. 
Figura 3 - Classificação e categorização dos PPGAs por eixos curriculares.

\begin{tabular}{|l|l|}
\hline FGV/RJ (4) 2 Organizz 1 Método: & 3 Finanças \\
\hline
\end{tabular} UFRGS (441 1 Organiż 2 Método: 2 Market PUC/RJ(441 Organizz 2 Método:2 UFMG (43 1 Organizz 2 Método 2 Marketi UFRJ (43a) 2 Organiz= 1 Método 1 Mark FGV/SP (4 1 Organiza 2 Método: 1 Market

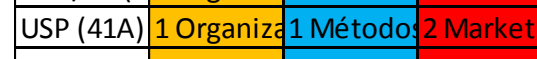
UFLA (41A 2 Organiza 1 Método 3 Marke UNB (40A) 1 Organiza 2 Método: UFPB/JP ( 2 Organizz 2 Método 1 UFRN (38:2 Organiz 1 Método: UFSC (38a 1 Organiza 2 Método: \begin{tabular}{|l|l|l|}
\hline PUC/SP (3 1 Organiza \\
\hline
\end{tabular} UFBA (33a 1 Organizz 2 Método UFPR (24a) 2 Organiza 1 Método 1 UFPE (21a 1 Organiz 3 Método 1 FURB (19A) 2 Organiza 1 Método UPM (17A 1 Organiza

UNISINOS 1 Organiz 1 Método 2 Market PUC/PR(1)

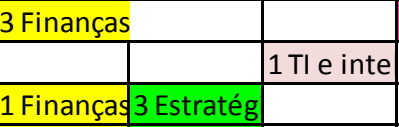
1 Finanças 3 Estratég

1 Finanças 3 Estratég $3 \mathrm{TI}$, gestã 1 Finanças 1 Estratég 1 Tl e inte 1 Finanças 2 Estratég 1 Tl e inte

UFES (16A) 10 Tganiz $2 \mathrm{~N}$ UDESC (161 Organize

UNIVALI () 1 Organiz 2 Método USCS (13A 2 Organiza UFSM (13A1 Organiz 2 Método UECE (11A 1 Organizz 1 Método \begin{tabular}{|l|l|}
\hline UFV (11A) & 2 Método \\
\hline
\end{tabular} UNINOVE 3 Organize 1 Método: 1 PUC/RS (1 3 Método \begin{tabular}{l|l} 
UCS (10A) 1 Organiza 3 Método \\
\hline
\end{tabular} UNIR (10A) 2 Organiza 1 Método FEI (9A) 1 Método: 2 Market UNIGRAN 1 Organiza 2 Método \begin{tabular}{|l|l|}
\hline UFMS (8A) & 1 Método \\
\hline UNAMA & \\
\hline
\end{tabular} UNAMA ( 22 Organizz 2 Método \begin{tabular}{|l|l|}
\hline UEM (6A) 1 Organiza \\
\hline
\end{tabular} $\operatorname{ESPM}(6 \mathrm{~A})$

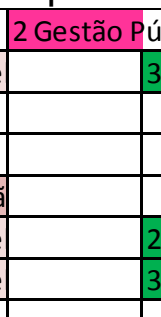

2 Finanças

i| 3 Finanças 3 Estratég $2 \mathrm{TI}$, gestã

\title{
2 Finança 3 Estratég $2 \pi$, gestá
}

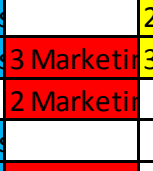

3 Finanças $3 \mathrm{TI}$, gestã

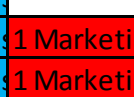

\begin{tabular}{l|l|l|} 
& 3 Estratég & \\
& & \\
\hline
\end{tabular}

2 Estratég $2 \mathrm{TI}$, gestã

\begin{abstract}
2 Finanças 1 Estratég $2 \mathrm{Tl}$, gestã
\end{abstract}

3 Sustentab. e Gestão Social
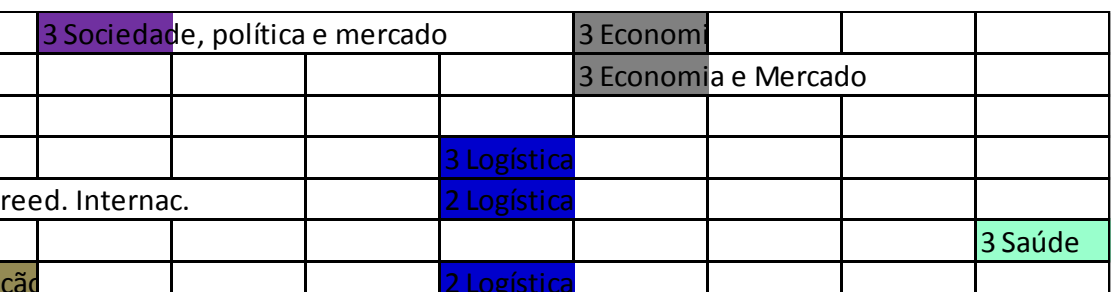

1 Fing 2 Estratég

1 Finanças 2 Estratég

til 3 Finanças 1 Estraté

Regae: Rev. Gest. Aval. Educ.

Santa Maria

\begin{tabular}{|c|c|}
\hline 1 Estratég & \\
\hline & \\
\hline 2 Estratég & \\
\hline & \\
\hline & \\
\hline & \\
\hline 1 Estratég & \\
\hline \begin{tabular}{|l|l|}
3 Estratég & 1 \\
\end{tabular} & 1 TI, gestã \\
\hline \begin{tabular}{|l|l}
2 Estratég & 1 \\
\end{tabular} & $1 \mathrm{TI}$, gestã \\
\hline 3 Estratég & \\
\hline 2 Fstratég & \\
\hline 3 Estratég & \\
\hline 2 Estratég & \\
\hline 1 Estratég & \\
\hline 3 Estratég & \\
\hline
\end{tabular}

\begin{tabular}{|l|l}
\hline & \\
& \\
& 1 Gestão \\
& \\
\hline & \\
\hline & 1 Gestã \\
\hline stã & \\
\hline & \\
\hline & \\
\hline
\end{tabular}

\begin{tabular}{|c|c|}
\hline & 2 Negócio \\
\hline 2 Sustent? & \\
\hline 3 Sustent & 2 Negócio \\
\hline
\end{tabular}

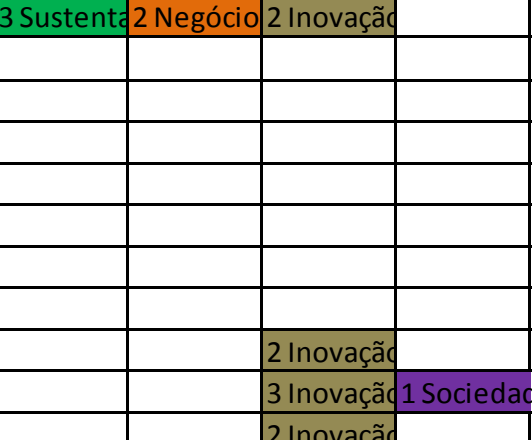

\begin{tabular}{|lll|l|l|l|l|l|}
\hline & & 2 Logistica & & & \\
\hline & & & & Economia & & & \\
\hline & & & & & & \\
\hline & & & & & & \\
\hline
\end{tabular}

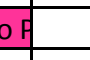

\begin{tabular}{|l|l|}
\hline & \\
\hline & \\
\hline & \\
\hline
\end{tabular}

\begin{tabular}{|l|l|l|l|l|}
\hline & & 2 Inovaçãa & & \\
\hline & & & & \\
\hline & 2 Sustentácio & & & \\
\hline & & & & \\
\hline
\end{tabular}

\begin{tabular}{|c|c|}
\hline & 2 Inovaçã \\
\hline & \\
\hline & \\
\hline & \\
\hline & \\
\hline & \\
\hline & 1 Inovaçã \\
\hline & \\
\hline 2 Sustent & \\
\hline 1 Sustent & \\
\hline 1 Sustent & 2 Inovaçã \\
\hline & \\
\hline 2 Sustent & \\
\hline 1 Sustent & \\
\hline & \\
\hline & 2 Inovaçã \\
\hline
\end{tabular}
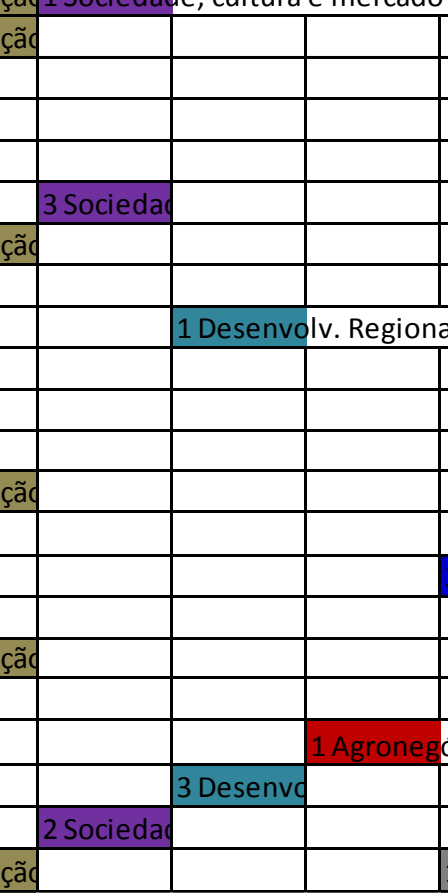

2021 
Agrupamentos formados pelos PPGAs

A partir da classificação fornecida pelos filtros os 37 programas pesquisados foram organizados em cinco agrupamentos, conforme a quantidade de eixos que possuem em comum com outros programas, sendo iniciado com a quantidade de seis eixos comuns, passando posteriormente para cinco, quatro e assim sucessivamente.

O primeiro grupo é também o mais homogêneo, conforme ilustrado na figura 4. É formado pelos programas da UFRJ, USP, FGV/SP, UFMG, UFPR, UFPE e UCS, que possuem entre si similaridade em mais de seis eixos, demonstrando forte grau isomórfico, especialmente os programas da USP e UFRJ, que possuem oito eixos semelhantes. A similaridade em seis eixos leva a crer que há forte semelhança estrutural, uma vez que as disciplinas estão alinhadas com a expertise do corpo docente, com as linhas de pesquisa e com a estrutura física e tecnológica e refletem os acordos e parcerias interinstitucionais nacionais e internacionais dos programas (Capes, 2016; Segev; Raveh; Farjoun, 1999). Neste grupo encontram-se três dos quatro programas melhor conceituados de acordo com a avaliação trienal Capes 2013, sendo USP, conceito 7, FGV/SP, conceito 7, e UFMG, conceito 6 . O programa da UFRJ possui conceito 5 e possuem conceito 4 os PPGAs da UFPE, UFPR e UCS. Nesse agrupamento é possível notar elevada heterogeneidade quanto aos conceitos.

Destaca-se neste cenário os currículos da UFPR, UCS e UFPE por serem tão similares aos cursos mais antigos a ponto de o PPGA da UFPE ter sete eixos semelhantes à USP. Os programas da UCS e UFPR possuem seis eixos em comum com a USP, sendo que a UCS possui os sies principais alinhados perfeitamente com os eixos que compõem a grade da USP. Os demais PPGAs são contemporâneos, o que pode explicar a similaridade curricular pelo período de fundação (Dacin, 1997), já os programas mais jovens despertam curiosidade quanto à decisão de aproximar tanto sua estrutura dos programas mais antigos.

O grupo 2, conforme figura 4, é formado por todos os cursos que apresentaram similaridade com os programas do Grupo 1, da ordem de cinco eixos em comum. Portanto, esses programas possuem estruturas curriculares bastante próximas do Grupo $1 \mathrm{com}$ algum grau de diferenciação, demonstrando que há forte isomorfismo estrutural com o grupo principal. As características dos componentes desse grupo é que são bastante variados em termos de idade, incluindo programas bastante antigos como os da PUC/RJ e Ufrgs, que foram os segundos PPGAs criados no Brasil, ao mesmo tempo em que abarca o programa da ESPM, o mais jovem do país e PPGAs de idade intermediária, como PUC/PR, Unisinos e Uninove, demonstrando que as decisões quanto à inspiração dos currículos não estão relacionadas com o período de fundação do programa, mas talvez esteja alinhado com a decisão de atualização e esforço para adaptação ao ambiente, ou talvez ainda, adaptação às incertezas ambientais. Todos os PPGAs deste grupo possuem conceito 5, com exceção da UFSC e ESPM, que possuem conceito 4 .

$O$ terceiro grupo representa os programas que possuem quatro eixos em comum com os PPGAs do primeiro e segundo grupos, conforme figura 4. A similaridade do grupo é razoavelmente alta com os componentes do Grupo 1 e alguns do Grupo 2, em especial - PPGA da Ufrgs, no total de quatro eixos em comum com os programas dos grupos anteriores. Os programas que possuem similaridade de quatro eixos com os PPGAs do Grupo 1 são Unir, Furb, PUC/RS, Univali, Unama, FEl e UEM. A grade dos programas da 
UFLA e UFSM são semelhantes à grade curricular da UFRGS. Alguns programas incluem similaridades com outros PPGAs além dos componentes dos Grupos 1 e 2, principalmente os mais jovens que tendem a espelhar seus currículos em outros programas igualmente jovens. Um dos exemplos é o da Unama, que mantém similaridade também com Unir e UCS. Quanto aos conceitos todos os cursos possuem conceito 4, exceto Ufla, PUC/RS e Univali, que possuem conceito 5 na avaliação Capes 2013.

O grupo 4 não está totalmente ligado à sequência de eixos pela qual foram obtidos 0 filtro 1 e 2, demonstrando fraca relação de intensidade da similaridade de eixos com os programas que compõem os grupos anteriores. Embora todos os programas deste grupo possuam três eixos alinhados com o currículo dos PPGAs do Grupo 1 possuem similaridades com outros programas mais jovens e características próprias, como currículos enxutos e eixos exclusivos, sendo exemplo de eixos diferenciados os PPGAs da USCS e UFMS. Todos os programas possuem conceito 4, com exceção da UPM e USCS, cujo conceito é 5 .

Existe um outro grupo capitaneado pela FGV/RJ, que possui currículos similar, com maior ou menor grau de proximidade. O principal eixo que une o grupo é voltado para a área da Gestão Pública, embora existam outros eixos comuns. O único programa com grade ampla do grupo é o da FGV/RJ, os demais possuem currículos reduzidos e todos os PPGAs possuem $100 \%$ de similaridade dos eixos com os eixos que compõem o currículo da FGV/RJ. Em termos de conceitos, assim como o primeiro, esse é um grupo bastante heterogêneo, possuindo conceitos 6, FGV/RJ, 5, UNB e UFBA, e 4: UFV, Ufes e Udesc.

O programa da UFRN também poderia fazer parte desse agrupamento, pois possui quatro dos seis eixos semelhantes com a FGV/RJ e demais componentes, entretanto, já foi classificado no grupo dos que possuem 5 eixos em comum com os PPGAs do primeiro grupo, tornando evidente que este programa procurou manter equilíbrio, tanto com os PPGAs mais tradicionais em termos de currículos comuns, quanto com o programa mais antigo do Brasil, que possui vocação para os estudos da área pública.

Os grupos foram organizados em função do nível de intensidade quanto à similaridade de seus currículos. Sendo assim, o primeiro grupo representa o maior nível de similaridade, evoluindo para o nível de menor intensidade possível, representado pelo Grupo 4, no qual é possível perceber maior nível de diferenciação. O mesmo se verifica no grupo 5, no qual os currículos dos seus programas divergem de modo a se tornar independente dos demais. A intensidade de influência e divisão dos grupos pode ser visualizada na figura 4 . 
Figura 4 -

Escala de similaridade dos agrupamentos formados pelos PPGAs.
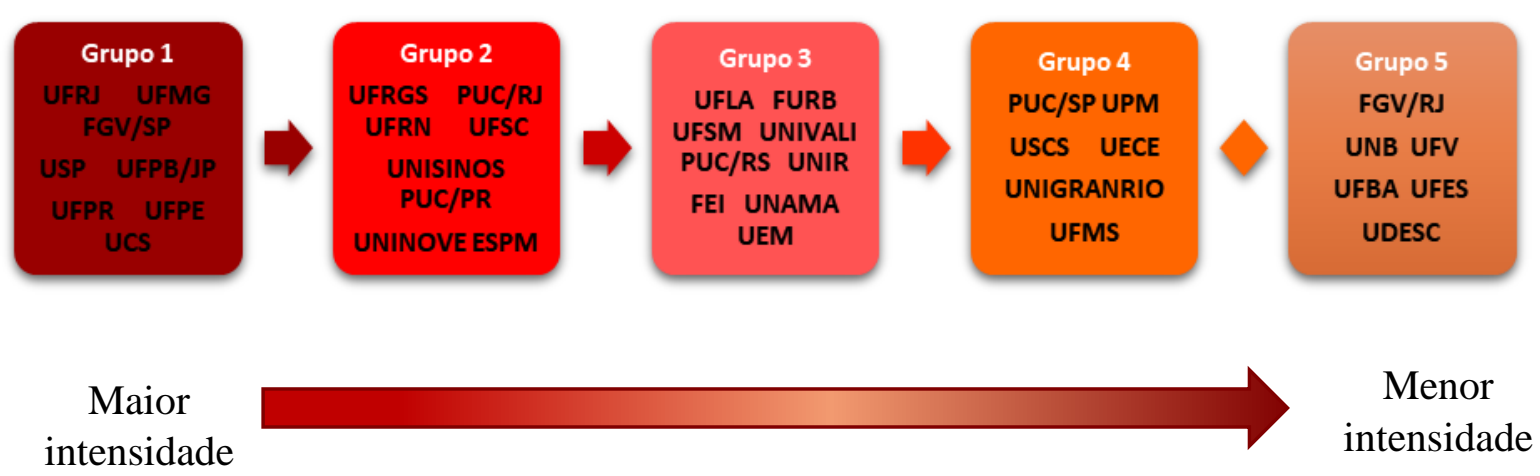

Fonte: dados da pesquisa.

Os grupos em que se nota maior intensidade de similaridade indicam maior nível de isomorfismo, de modo que existe maior semelhança estrutural entre eles, ao passo que nos dois últimos grupos é possível inferir que as decisões estruturais foram direcionadas para a diferenciação dos currículos em relação aos programas tradicionais, que compõem os grupos mais isomórficos. Apesar de existir isomorfismo entre todos os componentes dos cinco grupos, há diferenças significativas quanto às intensidades isomórficas entre eles.

\section{Discussão dos resultados}

Utilizando como método a categorização em eixos temáticos e classificação dos componentes curriculares de todos os PPGAs de conceito 4, 5, 6 e 7 na avaliação da Capes, foi possível verificar o nível de similaridade entre os programas, que no caso de alguns oscila entre 80 e $90 \%$, ou seja, os resultados demonstraram que existe isomorfismo entre os currículos dos PPGAs. Contudo, as similaridades não se restringem apenas aos currículos porque, segundo a Capes (2016), as disciplinas devem estar alinhadas com as linhas de pesquisa e com as áreas de concentração dos programas que, por sua vez, demandam um corpo docente com as competências relacionadas, além de uma série de recursos físicos e tecnológicos para o seu desenvolvimento. Assim, os currículos representam uma gama de recursos associados que tornam evidente 0 isomorfismo mimético, confirmando, portanto, a primeira proposição do estudo.

A partir das análises individuais dos PPGAs e dos agrupamentos formados foi possível constatar semelhanças entre os componentes de cada grupo e diferenciação entre os diversos grupos formados, confirmando a segunda proposição. Os grupos 1, 2 e 3 apresentaram características mais homogêneas quanto à similaridade dos currículos, variando em termos de intensidade, ao passo que os grupos 4 e 5 se distanciaram dos demais agrupamentos. Os dois últimos grupos demonstraram independência em relação aos demais, indicando que as decisões para a diferenciação estrutural podem ter prevalecido sobre as decisões para a conformidade, embora a quantidade de programas que compõem esses grupos seja minoria se comparados ao total pesquisado. Outro fator 
que pode ter colaborado para a configuração das propostas dos programas pode ser as competências do corpo docente, já que a Capes avalia o alinhamento entre a proposta do programa e a produção científica do corpo docente permanente, embora exista a possibilidade de contratar os professores coerentes com a proposta.

A maioria dos PPGAs optou pela similaridade, indicando que a prática de inspiração em grades curriculares consolidadas é uma estratégia amplamente utilizada (Pedersen et al. 2013). O contexto educacional dos PPGAs aponta que a similaridade entre os currículos, que representa a configuração dos demais recursos, indica que a decisão de se espelhar no modelo de outras organizações pode ser estratégica ou reflexo da regulamentação, pois apesar de as avaliações terem se iniciado em meados de 1970, a Capes exerceu diferentes intensidades regulatórias ao longo do tempo, vindo a adotar uma postura mais rígida somente a partir da década de 1990 (Maccari et al., 2014; Spagnolo; Calhau, 2002; Sguissardi, 2006; Morosini, 2001), o que pode ter levado a influenciar diferentes comportamentos das instituições (Dacin, 1997).

O ponto de convergência entre os dois tipos de comportamentos é defendido por (Pedersen et al. 2013), que afirma que as respostas das instituições podem ser o produto das pressões institucionais, isso porque, por melhor estruturada e provida de estratégia que seja a organização, o não cumprimento das normas regulatórias, ocasiona sanções e penalidades. A maior penalidade imposta a uma instituição no ambiente organizacional das pós-graduações é a atribuição de conceitos inferiores, pois isso se reflete diretamente na captação de recursos (Dias Sobrinho, 2003) junto às agências de financiamento do ensino superior e fomento à pesquisa, assim como na legitimação. Esse fator pode levar muitas instituições a colocarem as normas regulatórias acima dos próprios objetivos estratégicos (Maccari et al., 2014; Spagnolo; Calhau, 2002).

Dacin (1997) já havia identificado a força das normas institucionais sobre as decisões das organizações em sua época de fundação. No caso da presente pesquisa isso é possível de ser verificado no caso dos PPGAs mais jovens do estudo, que exibem características como currículos mais enxutos, que remete a corpos docentes mais reduzidos e menor quantidade de linhas de pesquisa. O comportamento dos programas mais jovens pode ser reflexo das normas difundidas pela Capes nos últimos anos, pois quando o Estado domina o ambiente social, as estruturas das organizações se tornam cada vez mais homogêneas e refletem os rituais de normas e regras institucionalizadas e legitimadas pelas instituições reguladoras (Meyer; Rowan, 1977). Assim, as organizações que melhor se adaptam ao contexto (Dimaggio; Powell, 1983) obtém os melhores resultados.

Quanto à terceira proposição, que sugeria que o isomorfismo estrutural poderia se refletir no desempenho do PPGA, o que se observou é que os conceitos dos PPGAs componentes de cada agrupamento são diversificados. Todos os grupos apresentaram conceitos variados entre os seus componentes sendo quena maioria dos casos, os cursos mais jovens possuem conceitos menores e os programas antigos possuem conceitos maiores, demonstrando que o conceito tem uma correlação positiva mais intensa com a idade do que com a similaridade estrutural. Nesse caso é provável que a influência americana sobre a Administração (Santos; Azevedo, 2009), conduziram os programas 
mais antigos a adotar estruturas curriculares semelhantes, o que Ihes deu certa vantagem pela trajetória de dependência (Karim; Mitchell, 2000; Bernardi, 2012), além do desenvolvimento de competências essenciais que seriam difíceis de serem obtidas pelos programas mais jovens (Prahalad; Hamel, 1990).

O papel avaliativo regulador da Capes pode ter contribuído para o isomorfismo, entretanto, essa mesma instituição tem adotado medidas para evitar a continuidade da propagação isomórfica, de modo que nos últimos anos passou a valorizar as propostas com área de concentração e linhas de pesquisa inovadoras nas propostas de novos cursos (Capes, 2016). O fenômeno pode ser compreendido pela verificação das mudanças dos modelos de avaliação e formas de atuação da Capes ao longo dos anos. Se, por um lado, a agência reguladora contribuiu como um dos maiores vetores para o isomorfismo, por outro, se posicionou como o agente mitigador das similaridades estruturais no campo científico das pós-graduações, a fim de evitar a saturação da área.

Considerando que o objetivo da Capes é manter um sistema de avaliação aprimorado para a obtenção de um padrão nacional de excelência acadêmica (Capes, 2008), é certo que atuará como incentivadora para que os programas busquem outras fontes como modelo de inspiração na construção de suas propostas, principalmente modelos internacionais, que alinharia a academia científica brasileira com os temas de interesse de pesquisa em nível mundial.

Apesar dessa prática também receber críticas como as de Rossoni e de São Pedro Filho (2015), que veem com preocupação a cópia de matrizes internacionais para o contexto brasileiro sob o risco de não se adequarem à realidade nacional e gerar problemas quanto à formação de profissionais mal qualificados para operar no mercado nacional, que possui particularidades próprias, no caso das pós-graduações pode ser positivo. Considerando que a ciência é universal e que a globalização demanda atualização constante, a inspiração em modelos internacionais pode ser um ponto positivo para o desenvolvimento da ciência no país.

Por outro lado, as maiores implicações do isomorfismo num campo organizacional é a geração de entraves para os avanços qualitativos da produção científica. A produção científica do corpo docente e discente de um programa tem estreita ligação com a matriz curricular, porque reflete suas linhas de pesquisa, área de concentração e competências do corpo docente, e, esse alinhamento é avaliado pela Capes em relação à proposta do programa. Portanto, o alinhamento internalizado de uma área científica pode significar lapsos qualitativos na ciência nacional (Volpato; Freitas, 2003).

Em suma, os resultados demonstraram que há maior grau de isomorfismo entre os grupos mais homogêneos (Dimaggio; Powell, 1983), tornando evidente a disseminação da prática de espelhamento nas propostas curriculares, em especial dos programas menos tradicionais para os mais legitimados pela sociedade ou pelos órgãos reguladores (Meyer; Rowan, 1977; Deephouse, 1996; Almeida; Callado, 2017). Situação semelhante já havia sido constatada por Dale (2004) ao encontrar uniformização curricular a nível mundial.

Outros autores também investigaram a ação da regulamentação sobre as instituições de ensino superior e destacaram que houve uma movimentação mundial, nos últimos 25 anos, de disseminação de regimes de garantia da qualidade em mais da metade dos países do globo, de modo que os governos implantaram sistemas de certificação e conformidade por meio da validação de padrões de qualidade (Jarvis, 2014;

\begin{tabular}{|l|l|l|l|l|l|}
\hline Regae: Rev. Gest. Aval. Educ. & Santa Maria & v. 10 & n. 19 & e62676, p. 1-21 & 2021 \\
\hline
\end{tabular}


Croucher; WoeleRT, 2016), que geraram aumento de pressões combinadas com variáveis ambientais incertas, produzindo sobre as instituições de ensino superior tendências de convergência quanto à estrutura (Croucher; Woelert, 2016). No Brasil não foi diferente. Por meio da Capes o país ingressou na lógica global que, segundo Hammarfelt e Fredrik (2015), se trata do aumento da competitividade na educação superior de um mercado de pesquisa cada vez mais global.

\section{Considerações finais}

Nesse estudo nos propomos a identificar a presença de isomorfismo mimético a partir das similaridades estruturais dos currículos dos PPGAs, ao mesmo tempo em que procuramos verificar se a participação nos agrupamentos determinava seu posicionamento no ranking da Capes. Cinco agrupamentos foram identificados a partir da verificação da intensidade de similaridade entre os currículos curriculares dos PPGAs, sendo possível observar nível máximo de semelhança entre os programas que compuseram o primeiro agrupamento, passando pelos grupos intermediários, um grupo de fraca similaridade até o agrupamento com grade diferenciada em relação aos demais.

As contribuições do estudo estão relacionadas as discussões sobre a presença do isomorfismo nos PPGAs e o papel da Capes nesse contexto e no desenvolvimento científico brasileiro. A Capes, ao contrário do que parece, pode ter um papel importante na redução das estruturas isomórficas. Com os resultados encontrados foi possível observar tendência isomórfica entre os programas mais antigos e com as exigências impostas nos últimos anos quanto à inovação, os programas estão sendo forçados a incorporar as discussões que refletem o futuro da Administração. Isso não impede que os programas mais jovens assemelhem parte de seus currículos aos demais, mas os força a agregar eixos interdisciplinares, diversificados e inovadores. Assim sendo, apesar das críticas quanto às métricas de avaliação, a Capes pode estar contribuindo para a diversificação, atualização e modernização da ciência em nível nacional, ao incentivar os programas a promover intercâmbios de conhecimento com instituições de outros países, que possibilita $o$ alinhamento das pesquisas com os temas de interesse mundial.

As proposições sustentadas no estudo foram confirmadas total e parcialmente. A primeira proposição - $\mathrm{P} 1$-, que atestava a presença de isomorfismo entre os programas foi confirmada, sendo possível observar alto nível de similaridade entre boa parte dos currículos dos programas pesquisados, assim como foi possível identificar os principais programas que podem ter servido de referência para a construção da grade dos demais, sendo USP, FGV/SP, UFRJ, PUC/RJ, UFRGS e FGV/RJ. Da mesma forma a segunda proposição - P2 - foi confirmada quando constatamos diferentes agrupamentos de programas, que tornou evidente o grau de disposição ao se inspirar no currículo de outros PPGAs. Entretanto, é notório que a interferência do órgão regulador - Capes - pode ter sido decisiva na formatação do currículo, principalmente para os novos cursos, que surgiram depois do enrijecimento das normas. Mesmo assim foi possível constatar programas jovens com grades muito similares aos antigos.

Quanto à terceira proposição - P3 - em que se afirma que o pertencimento a um determinado grupo pode se refletir no desempenho do PPGA na avaliação da Capes, constatamos que a participação nos grupos não assegura a obtenção de resultados semelhantes aos demais componentes, tampouco conceitos mais elevados na avaliação, 
sendo que essa condição certamente depende das competências organizacionais, a trajetória de dependência, tradição, configuração de recursos e uma série de outras variáveis estratégicas não mensuradas nessa pesquisa, que proporcionariam aos programas produzir resultados satisfatórios e, ao mesmo tempo, se adaptar ao contexto institucional altamente regulamentado, para alcançar legitimidade junto à sociedade e aos órgãos reguladores.

As limitações do estudo se restringem à metodologia em com algumas etapas, nas quais foi necessário o uso da subjetividade como, por exemplo, a classificação das disciplinas e categorização dos eixos, todavia, o método se mostrou importante pelo fato das análises puramente objetivas não captarem a profundidade das relações, particularidades e características da população.

\section{Referências}

ALMEIDA, Karla Katiuscia Nóbrega de; CALLADO, Aldo Leonardo Cunha. Indicadores de desempenho ambiental e social de empresas do setor de energia elétrica brasileiro: uma análise realizada a partir da ótica da teoria institucional. Revista de Gestão, Finanças e Contabilidade, Senhor do Bonfim, v. 7, n.1, 2017.

APPOLD, Stephen. Locational patterns of US industrial research: mimetic isomorphism and the emergence of geographic charisma, Regional Studies. Falmer Brighton, 39, 2005, p.17-39.

BACKES, Danieli Artuzi Pes; SERRA, Fernando Antonio Ribeiro; NETO, Feris Antonio Zarour Identifying structural similarities between stricto sensu post-graduation programs in management regarding the strategy tripod. REGE Revista de Gestão, São Paulo, v. 25, n. 3, 2018, p. 303-320.

BARDIN, Laurence. Análise de conteúdo. Lisboa: Edições 70, 1977.

BENDERS, Jos; BATENBURG, Ronald; VAN DER BLONK, Heico. Sticking to standards; technical and other isomorphic pressures in deploying ERP-systems. Information \& Management, Amsterdam, v. 43, n. 2, 2006, p. 194-203.

BERNARDI, Bruno Boti. O conceito de dependência da trajetória (path dependence): definições e controvérsias teóricas. Perspectivas: Revista de Ciências Sociais, Araraquara, v. 41, 2012.

BROUTHERS, Lance Eliot; O'DONNELL, Edward; HADJIMARCOU, John. Generic product strategies for emerging market exports into triad nation markets: A mimetic isomorphism approach. Journal of Management Studies, Reino Unido, v. 42, n. 1, 2005, p. 225-245.

BURNS, Lawton Robert; WHOLEY, Douglas. Adoption and abandonment of matrix management programs: Effects of organizational characteristics and interorganizational networks. Academy of Management Journal, New York, v. 36, n. 1, 1993, p. 106-138.

CAPES. Cursos avaliados e reconhecidos. Brasília: Capes, 2018b. Disponível em https://sucupira.capes.gov.br/sucupira/public/consultas/coleta/programa/quantitativos/quan titativoAreaAvaliacao.jsf. Acesso em 15 jan. 2018. 
CAPES. Documento de área: Administração Pública e de Empresas, Ciências Contábeis e Turismo 2016. Brasília: Capes, 2018a. Disponível em http://www.capes.gov.br/images/documentos/Documentos_de_area_2017/27_ADMI_doca rea_2016.pdf. Acesso em 15 jan. 2018.

CAPES. História e missão. Brasília: Capes, 2008. Disponível em http://www.capes.gov.br/historia-e-missao. Acesso em 21 fev. 2020.

CAPES. Orientações para apcn-2016. Brasília: Capes, 2016. Disponível em https://www.capes.gov.br/images/documentos/Criterios_apcn_2016/Criterios_APCN_Admi nistracao.pdf. Acesso em 20 jul. 2020.

CAPES. Plataforma Sucupira. Brasília: Capes, 2017a. Disponível em https://sucupira.capes.gov.br/sucupira/public/consultas/coleta/disciplina/listaDisciplina.jsf;j sessionid=VyKGEDdY9EGrj5vgsYcydDsS.sucupira-203. Acesso em 19 fev. 2020.

CAPES. Portaria n. 59, de 21 de março de 2017: regulamento da avaliação quadrienal. Brasília: Capes, 2017. Disponível em http://capes.gov.br/images/stories/download/avaliacao/27032017-Portaria-59-21-03-2017Regulamento-da-Avaliacao-Quadrienal.pdf. Acesso em 19 jun. 2020.

CAPES. Sobre avaliação de cursos - 2015. Brasília: Capes, 2015. Disponível em http://www.capes.gov.br/acessoainformacao/perguntas-frequentes/avaliacao-da-posgraduacao/7421-sobre-avaliacao-de-cursos. Acesso em 9 jul. 2020.

CROUCHER, Gwilym; WOELERT, Peter. Institutional isomorphism and the creation of the unified national system of higher education in Australia: an empirical analysis. Higher Education, Dordrecht, v. 71, n. 4, 2016, p. 439-453.

DACIN, Tina. Isomorphism in context: the power and prescription of institutional norms. Academy of Management Journal, New York, v. 40, n. 1, 1997, p. 46-81.

DALE, Roger. Globalização e educação: demonstrando a existência de uma cultura educacional mundial comum. Educação \& Sociedade, Campinas, v. 25, n. 87, 2004.

DARUS, Faizah; HAMZAH, Engku Artini Che Ku; YUSOFF, Haslinda. CSR web reporting: The influence of ownership structure and mimetic isomorphism. Procedia Economics and Finance, Amsterdam, v. 7, 2013, p. 236-242.

DEEPHOUSE, David. Does isomorphism legitimate? The Academy of Management Journal, New York, v. 39, n. 4, 1996, p. 1024-1039.

DIAS SOBRINHO, José. Avaliação da educação superior regulação e emancipação. Avaliação, Sorocaba, v. 8, n. 2, 2003, p. 31-47.

DIMAGGIO, Paul; POWELL, Walter. The iron cage revisited: institutional isomorphism and collective rationality in organizational fields. American Sociologic Review, Washington, v. 48, n. 2, 1983, p. 147-160.

FRUMKIN, Peter; GALASKIEWICZ, Joseph. Institutional isomorphism and public sector organizations. Journal of Public Administration Research and Theory, Oxford, v. 14, n. 3, 2004, p. 283-307.

HAIR JR., Joseph F; ANDERSON, Rolph. E.; TATHAM, Ronald L.; BLACK, William C. Análise multivariada de dados. Porto Alegre: Bookman, 2005.

HAMMARFELT, Björn; FREDRIK, Astrom. The multi-layered and multilevel use of bibliometric measures in Swedish universities: Isomorphism, translation and strategic choice. INTERNATIONAL CONFERENCE ON SCIENCE AND TECHNOLOGY INDICATORS, 20, 2015. Anais ... Lugano: STI, 2015. 
HAN, Shin-Kap. Mimetic Isomorphism and Its Effect on the Audit Services Market. Social Forces, North Carolina, v. 73, n. 2, 1994, p. 637-664.

HAVEMAN, Heather. Follow the Leader: Mimetic Isomorphism and Entry Into New Markets. Administrative Science Quarterly, Ithaca, v. 38, n. 4, 1993, p. 593-627.

INEP. Censo da educação superior 2016: principais resultados. Brasília: Inep, 2018. Disponível

http://download.inep.gov.br/educacao_superior/censo_superior/documentos/2016/censo_s uperior_tabelas.pdf. Acesso em 14 jan. 2018.

JARVIS, Darryl S. Policy transfer, neo-liberalism or coercive institutional isomorphism? Explaining the emergence of a regulatory regime for quality assurance in the Hong Kong higher education sector. Policy and Society, Singapura, v. 33, n. 3, 2014, p. 237-252.

KARIM, Samira; MITCHELL, Will. Path-dependent and path-breaking change: Reconfiguring business resources following acquisitions in the US medical sector, 19781995. Strategic Management Journal, Chicago, 2000, p.1061-1081.

KNOKE, David. The spread of municipal reform: Temporal, spatial, and social dynamics. American Journal of Sociology, Chicago, v. 87, n. 6, 1982, p.1314-1339.

LANGRAFE, Taiguara de Freitas; BOAVENTURA, João Maurício Gama; SILVA, Ralph dos Santos; DA SILVA, Dirceu. Grupos estratégicos: um estudo dos cursos de graduação em Administração na cidade de São Paulo. RIAE. Revista Ibero-Americana de Estratégia, São Paulo, v. 8, n. 1, 2009, p. 78-101.

LU, Jane W. Intra-and inter-organizational imitative behavior: Institutional influences on Japanese firms' entry mode choice. Journal of International Business Studies, London, v. 33, n. 1, 2002, p.19-37.

MACCARI, Émerson Antonio; DE ALMEIDA, Martinho Isnard Ribeiro; RICCIO, Edson Luiz; ALEJANDRO, Thomas Brashear. Proposta de um modelo de gestão de programas de pós-graduação na área de Administração a partir dos sistemas de avaliação do Brasil (Capes) e dos Estados Unidos (AACSB). Revista de Administração, São Paulo, v. 49, n. 2, 2014, p. 280-290.

MACCARI, Émerson Antonio; LIMA, Manolita Correia; RICCIO, Edson Luiz. Uso do sistema de avaliação da Capes por programas de pós-graduação em Administração no Brasil. Revista de Ciências da Administração, Florianópolis, v. 11, n. 25, 2009.

MARTÍNEZ-FERRERO, Jennifer; GARCÍA-SÁNCHEZ, Isabel Maria. Coercive, normative and mimetic isomorphism as determinants of the voluntary assurance of sustainability reports. International Business Review, London, v. 26, n.1, 2017, p. 102-118.

MEYER, John. W; ROWAN, Brian. Institutionalized organizations: Formal structure as myth and ceremony. American Journal of Sociology, Chicago, v. 83, n. 2, 1977, p. 340363.

MOROSINI, Marilia Costa. Qualidade da educação universitária: isomorfismo, diversidade e eqüidade. Interface Comunicação, Saúde, Educação, Botucatu, v. 5, n. 9, 2001, p. 89102.

NASCIMENTO, Luis Felipe. Modelo Capes de avaliação: quais as consequências para o triênio 2010-2012? Administração: Ensino e Pesquisa, São Paulo, v. 11, n. 4, 2010, p. 579-600.

PECl, Alketa. A nova teoria institucional em estudos organizacionais: uma abordagem crítica. Cadernos Ebape, Rio de Janeiro, v. 4, n. 1, 2006, p. 1-12. 
PEDERSEN, Esben Rahbek Gjerdrum; NEERGAARD, Peter; PEDERSEN, Janni Thengaard Pedersen; GWOZDZ, Wencke. Conformance and deviance: company responses to institutional pressures for corporate social responsibility reporting. Business Strategy and the Environment, Bangkok, v. 22, n. 6, 2013, p. 357-373.

PRAHALAD, Coimbatore Krishnarao; HAMEL, Gary. The core competence of the corporation. Boston (Ma), 1990, p. 235-256.

QUIVY, Raymond; VAN CAMPENHOUDT, Luc. Manual de investigação em ciências sociais. Lisboa: Universidade Nova de Lisboa, 1998.

ROSSONI, Estela Pitwak; SÃO PEDRO FILHO, Flavio de. Riscos do isomorfismo mimético, a consciência crítica e o comprometimento ético de docentes e pesquisadores. InterSciencePlace, Santiago, v. 1, n. 18, 2015.

SANTOS, Ana Lucia Félix dos; AZEVEDO, Janete Maria Lins de. A pós-graduação no Brasil, a pesquisa em educação e os estudos sobre a política educacional: os contornos da constituição de um campo acadêmico. Revista Brasileira de Educação, Rio de Janeiro, v. 14, n. 42, 2009, p. 534-550.

SAVITRI, Rizky Ayu; FANANI, Zaenal. The institutionalization of accrual accounting: the perspective of new institutional sociology theory. Journal Dinamika Akuntansi, Semarang, v. 9, n. 2, 2017, p. 100-109.

SCAFUTO, Isabel Cristina; BACKES, Danieli Artuzi Pes; MACCARI, Emerson Grupos estratégicos isomórficos: um estudo com os cursos Master of Business Administration do ranking da América Economía. Revista de Ciências da Administração, Florianópolis, v. 19, n. 48, 2017, p. 136-149.

SEGEV, Eli, RAVEH, Adi; FARJOUN, Moshe. Conceptual maps of the leading MBA programs in the United States: core courses, concentration areas, and the ranking of the school. Strategic Management Journal, Chicago, v. 20, n. 6, 1999, p. 549-565.

SGUISSARDI, Valdemar. A avaliação defensiva no modelo Capes de avaliação: é possível conciliar avaliação educativa com processos de regulação e controle do Estado? Perspectiva, Piracicaba, v. 24, n. 1, 2006, p. 49-88.

SOUZA, Saulo Rodrigo Alves de; GUERRA, Maria das Graças Gonçalves Vieira. Avaliação da Educação Superior no Brasil. Regae: Rev. Gest. Aval. Educ., Santa Maria, v. 9, n. 18, 2020.

SPAGNOLO, Fernando; CALHAU, Maria Gladis. Observadores internacionais avaliam a avaliação da Capes. Infocapes-Boletim Informativo da Capes, v. 10, n. 1, 2002, p. 7-34.

TINGLING, Peter; PARENT, Michael. Mimetic isomorphism and technology evaluation: does imitation transcend judgment? Journal of the Association for Information Systems, Atlanta, v. 3, n. 1, 2002.

TOLBERT, Pamela S; ZUCKER, Lynne G. Institutional sources of change in the formal structure of organizations: the diffusion of civil service reform, 1880-1935. Administrative Science Quarterly, 1983, p. 22-39.

VOLPATO, Gilson Luiz; FREITAS, Eliane Gonçalves de. Challenge in scientific publication. Pesquisa Odontológica Brasileira, São Paulo, v. 17, 2003, p. 49-56.

YANG, Monica; HYLAND, MaryAnne. Re-examining mimetic isomorphism: similarity in mergers and acquisitions in the financial service industry. Management Decision, London, v. 50, n. 6,2012 , p. 1076-1095. 
Danieli Artuzi Pes Backes é professora na Faculdade de Administração e Ciências Contábeis da Universidade Federal de Mato Grosso.

Orcid: https://orcid.org/0000-0002-6411-6649.

Endereço: Avenida Fernando Corrêa da Costa, 2367 - 78060-900 - Cuiabá - MT Brasil.

E-mail: backes.dani@gmail.com.

Fernando Antonio Ribeiro Serra é professor no Programa de Pós-Graduação em Administração da Universidade Nove de Julho.

Orcid: https://orcid.org/0000-0002-8178-7313.

Endereço: Rua Deputado Salvador Julianelli, s/n - 05001-900 - São Paulo - SP Brasil.

E-mail: fernandorserra@gmail.com.

Josiane de Oliveira Lobato é gerente de avaliação institucional na Pró-Reitoria de Planejamento da Universidade Federal de Mato Grosso.

Orcid: https://orcid.org/0000-0002-4774-183X.

Endereço: Avenida Fernando Corrêa da Costa, 2367 - 78060-900 - Cuiabá - MT Brasil.

E-mail: josiane deoliveira@hotmail.com.

Sandra Negri é professora na Faculdade de Direito da Universidade Federal de Mato Grosso.

Orcid: https://orcid.org/0000-0002-3847-6456.

Endereço: Avenida Valdon Varjão, 6390 - 78607-059 - Barra do Garças - MT Brasil.

E-mail: sandra.negri.br@gmail.com.

Recebido em 5 de novembro de 2020.

Aceito em 7 de abril de 2021.

cc) (i) (8) $\ominus$ 